\title{
Hospital-based group medical quarantine increased the pre- dialysis blood pressure in maintenance hemodialysis patients
}

\author{
Yan Wang^, Liangying Gan, Li Zuo, Bing Yang, Ying Wang, Junqing Liang, Mengfan Ni \\ Department of Nephrology, Peking University People's Hospital, Beijing, China \\ Contributions: (I) Conception and design: All authors; (II) Administrative support: L Gan, L Zuo; (III) Provision of study materials or patients: All \\ authors; (IV) Collection and assembly of data: Y Wang, B Yang, Y Wang, J Liang, M Ni; (V) Data analysis and interpretation: Y Wang, L Gan; (VI) \\ Manuscript writing: All authors; (VII) Final approval of manuscript: All authors. \\ Correspondence to: Liangying Gan, MD. Department of Nephrology, Peking University People's Hospital, No. 11 Xizhimen South Street, Beijing \\ 100044, China. Email: ganl@bjmu.edu.cn.
}

\begin{abstract}
Backgrounda Quarantine is an effective measure to contain the spread of infectious disease, however, it can be a great challenge for patients undergoing maintenance hemodialysis (MHD). The purpose of this study was to evaluate the impact of hospital-based group medical quarantine (H-GMQ) on blood pressure (BP) in MHD patients, and the effect of age on change in BP.

Methods: MHD patients in our dialysis center who were under H-GMQ due to exposure to coronavirus disease 2019 (COVID-19) were enrolled. Their demographic data, clinical characteristics, and laboratory data were collected from 3 months before H-GMQ to the end of H-GMQ. They were divided into two groups by median age (61 y). BP and related data before and during H-GMQ between groups were analyzed. The association between age and change in BP was estimated using multivariable linear regression analysis.

Results: One hundred and thirty MHD patients were enrolled. The pre-dialysis systolic BP (SBP) and heart rate (HR) during H-GMQ were significantly higher than before, the serum sodium decreased significantly at the end of H-GMQ. After adjusting for covariates by multivariable regression, age had a negative correlation with $\mathrm{BP}$ elevation $\left(\mathrm{R}^{2}=0.218, \mathrm{P}=0.246\right)$. Subgroup analysis showed that both pre-dialysis SBP and diastolic BP (DBP) increased significantly during H-GMQ in patients less than 61 years old, instead, neither SBP nor DBP changed in the elderly.
\end{abstract}

Conclusions: H-GMQ increased the pre-dialysis BP in MHD patients, especially in younger patients. More attention should be paid to these patients' BP if another quarantine is needed.

Keywords: Blood pressure (BP); hospital-based group medical quarantine (H-GMQ); maintenance hemodialysis (MHD)

Submitted Jul 15, 2020. Accepted for publication Nov 03, 2020.

doi: 10.21037/apm-20-1421

View this article at: http://dx.doi.org/10.21037/apm-20-1421

\section{Introduction}

The coronavirus disease 2019 (COVID-19) outbreak has been developed as a pandemic and posed a significant threat to global health (1). Quarantine and isolation of patients and close contacts seem to be critical to prevent disease spread since no specific effective antiviral therapies have been identified until now.

In February 2020, a hemodialysis (HD) patient at Peking University People's Hospital was confirmed to have COVID-19, leading to hospital-based group medical quarantine (H-GMQ) of $143 \mathrm{HD}$ patients and 37 healthcare workers for 2 weeks.

H-GMQ can be a great challenge for patients with end-

^ ORCID: 0000-0003-2658-3526. 
stage kidney disease (ESKD). It was reported that they had higher risks of cardiovascular disease as well as other comorbidities, and their adjusted all-cause mortality rate was at least 10-fold higher than the non-ESKD population (2). The changes in living environment and lifestyle during H-GMQ may have an unexpected impact on them physically and psychologically. Moreover, younger patients and elderly patients differ in cardiovascular function and life experience, which may result in different BP changes during H-GMQ.

Although the prior severe acute respiratory syndrome (SARS; 2002-2003) and the Middle east respiratory syndrome (MERS; 2012-ongoing) outbreaks have spawned related research, little is known about the impact of $\mathrm{H}-\mathrm{GMQ}$ on patients' blood pressure (BP) when they were directly exposed to infectious disease outbreaks. Therefore, the purpose of this study was to investigate the impact of H-GMQ on HD patients' BP, and the relation between age and change in $\mathrm{BP}$.

We present the following article in accordance with the STROBE reporting checklist (available at http://dx.doi. org/10.21037/apm-20-1421).

\section{Methods}

\section{Study design and subjects}

This study was a prospective observational study conducted in a single HD center. A total of 143 HD patients of Peking University People's Hospital were under a 2-week H-GMQ from February 18, 2020 to March 2, 2020, after being exposed to COVID-19. These patients were screened for inclusion in this study. The inclusion criteria included patients who were on maintenance HD (MHD), defined as underwent HD for more than 3 months. The exclusion criteria included: (I) cardiovascular and cerebrovascular events within 3 months; (II) the active phase of rheumatic disease or uncontrolled malignancy within 3 months; (III) refused to give written consent.

Each of the patients was isolated in a separate room in a branch area of Peking University People's Hospital, they were not allowed to go outside unless dialysis. Shuttle buses were provided to commute patients between quarantine area and dialysis center, and they were required to wear masks during transportations and dialysis sessions. Nutrition diet was provided by the hospital, the principle of catering was low sodium, low potassium, low phosphorus, and low purine diet. Five $\mathrm{g} / \mathrm{d}$ of salt, which is $2 \mathrm{~g} / \mathrm{d}$ of sodium was included in the provided diet.

They were divided into two groups by median age. Their BPs and related clinical data before and during H-GMQ were collected and analyzed. Effect of age on BP change was examined.

This study was conducted in accordance with the Declaration of Helsinki (as revised in 2013). The study was approved by the Ethics Committee of Peking University People's Hospital (ethical approval number: 2020PHB04101 ), all patients provided informed written consent before study entry.

\section{Measurements of BP}

$\mathrm{BP}$ was measured in the non-access arm of patients using an oscillometric method. Pre-dialysis BP was measured after 5 minutes of rest before the start of dialysis, intra-dialytic BP was measured at 1-hour intervals during each dialysis session.

We examined 12 dialysis sessions per patient for 4 weeks, including the 2 weeks before H-GMQ and the 2 weeks of $\mathrm{H}-\mathrm{GMQ}$, and recorded BPs for each session.

The pre-dialysis mean arterial pressure (MAP) of each dialysis session was calculated using the following formula: $\mathrm{MAP}=[$ systolic BP $(\mathrm{SBP})+2 \times$ diastolic BP $(\mathrm{DBP})] / 3$.

Intra-dialytic SBP variability (SBPV), defined as the average of the differences between consecutive SBP measurements in each dialysis session, was calculated using the formula (3): $\mathrm{SBPV}=\frac{1}{N-1} \sum_{K-1}^{N-1}\left|S B P_{K+1}-S B P_{K}\right| . \mathrm{N}$ represents the number of $\mathrm{BP}$ measurements and $\mathrm{K}$ range from 1 to $\mathrm{N}-1, \mathrm{SBP}_{\mathrm{K}}$ is the SBP at measurement number $\mathrm{K}$.

The mean values of the 2 -week pre-dialysis $\mathrm{BP}$, predialysis MAP, and intra-dialytic SBPV before and during H-GMQ were calculated and compared.

\section{Demographic and clinical data}

Demographic and clinical characteristics were collected from medical records, including gender, age, body mass index (BMI), dialysis vintage, comorbidities, vascular access, and use of antihypertensive drugs.

The pre-dialysis heart rate (HR), inter-dialytic weight gain (IDWG), and ultrafiltration volume of each dialysis session from 2 weeks before $\mathrm{H}-\mathrm{GMQ}$ to the end of $\mathrm{H}-\mathrm{GMQ}$ were collected. IDWG rate (IDWGR) and ultrafiltration rate (UFR) was calculated respectively using the following formulas: IDWGR $=$ IDWG/dry weight $\times 100 \%$; UFR 


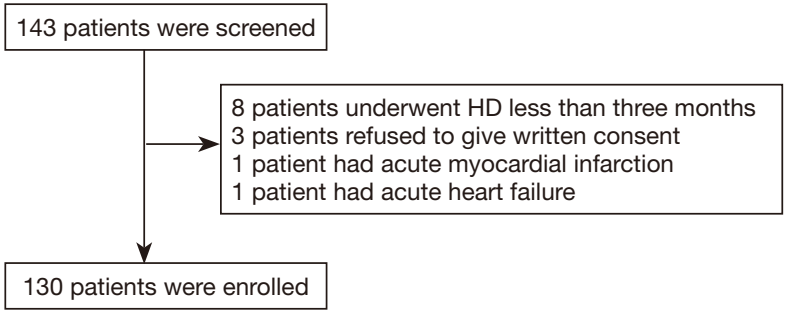

Figure 1 Enrollment of study subjects. HD, hemodialysis.

$=$ ultrafiltration volume/(dry weight $\times$ dialysis duration $)$. The mean values of the 2-week pre-dialysis HR, UFR, and inter-dialytic IDWGR before and during H-GMQ were calculated as baseline versus HCQ.

Laboratory data within 3 months before $\mathrm{H}-\mathrm{GMQ}$ were collected as the baseline, including hemoglobin, serum creatinine, albumin, adjusted calcium, phosphorus, sodium, potassium, and single-pool $\mathrm{Kt} / \mathrm{V}$. Electrolytes were tested at the end of H-GMQ for comparison.

All patients were assessed for mental health on the $12^{\text {th }}$ day in H-GMQ by using self-rating anxiety scale (SAS) and self-rating depression scale (SDS). The results were presented using index scores, which can be converted to by dividing the sum of the raw scores by 80 and multiplying by 100 (4). We also compared these to the patients' SAS and SDS scores within 3 months before H-GMQ.

\section{Dietary sodium intake assessment}

Dietary sodium intake was assessed by a 2-day diet diary, which consisted of one dialysis day and one non-dialysis day. Nutritional Data System for Research (NDSR, Minneapolis, MN, USA) software was used to calculate dietary sodium intake.

\section{Statistical analysis}

Normally distributed variables were expressed as mean \pm standard deviation (SD), non-normally distributed variables were expressed as median with the interquartile range (IQR) (Q25, Q75), categorical variables were expressed as frequencies and percentages. Differences in mean and median values before and during H-GMQ were compared using paired $t$-test. Effect of age on change in BP was examined using Pearson's correlation or Spearman's correlation (univariate analysis) and multivariate linear regression analysis, adjusted for covariates including gender, age, hypertension, diabetes, dialysis vintage, $\mathrm{BMI}$, difference in HR, IDWGR, serum sodium, serum potassium, SAS, and SDS. Continuous variables between groups stratified by age were compared using independent sample $t$-test, categorical variables between groups were compared using a chi-square test and Fisher exact test. A P value $<0.05$ was considered to be statistically significant. All statistical analyses were performed using SPSS 21.0 (IBM Corp., Armonk, NY, USA).

\section{Results}

One hundred and forty-three HD patients were screened and a total of 130 patients were enrolled. Thirteen patients were excluded due to short dialysis vintage, acute cardiovascular event, or refusal to participate in this study (shown in Figure 1). There were 85 males (65.4\%), with a median age of $61[48,71]$ years and a median dialysis vintage of $82.5(60.0,136.2)$ months. Among them 82 patients $(63.1 \%)$ had hypertension and 67 patients $(51.5 \%)$ were taking antihypertensive drugs. Additional baseline characteristics were shown in Table 1 .

During the H-GMQ period, no patient's antihypertensive drug prescription was modified, and the mean dietary sodium intake was $3.3 \mathrm{~g} / \mathrm{d}$. The pre-dialysis SBP, MAP, and HR of patients during H-GMQ were significantly higher than before, instead, the serum sodium decreased significantly compared with before. There were no significant differences in DBP, intra-dialytic SBPV, IDWGR, UFR and the SAS/SDS score between before and during H-GMQ, as shown in Table 2.

Univariate analysis revealed that the change in predialysis SBP had a positive correlation with hypertension $(\mathrm{P}=0.044)$ and a negative correlation with age $(\mathrm{P}=0.009)$. After adjusting for all the covariates in the multivariate linear regression model, SBP elevation was independently associated with age $\left(\mathrm{R}^{2}=0.218, \mathrm{P}=0.246\right)$. The younger the patients, the greater the elevation of pre-dialysis SBP during H-GMQ (Table 3).

Patients were then divided into two subgroups by median age to better evaluate the effect of age on BP. The baseline characteristics of the two groups were shown in Table 1. The younger group tended to have more males and higher BMI; the serum albumin, creatinine, and phosphorus levels were also higher compared with the elderly; the single-pool Kt/V was lower in the younger group but still reached the target $\mathrm{Kt} / \mathrm{V}$ recommended by Kidney Disease Outcomes Quality Initiative (KDOQI). 
Table 1 Baseline characteristics of patients stratified by median age

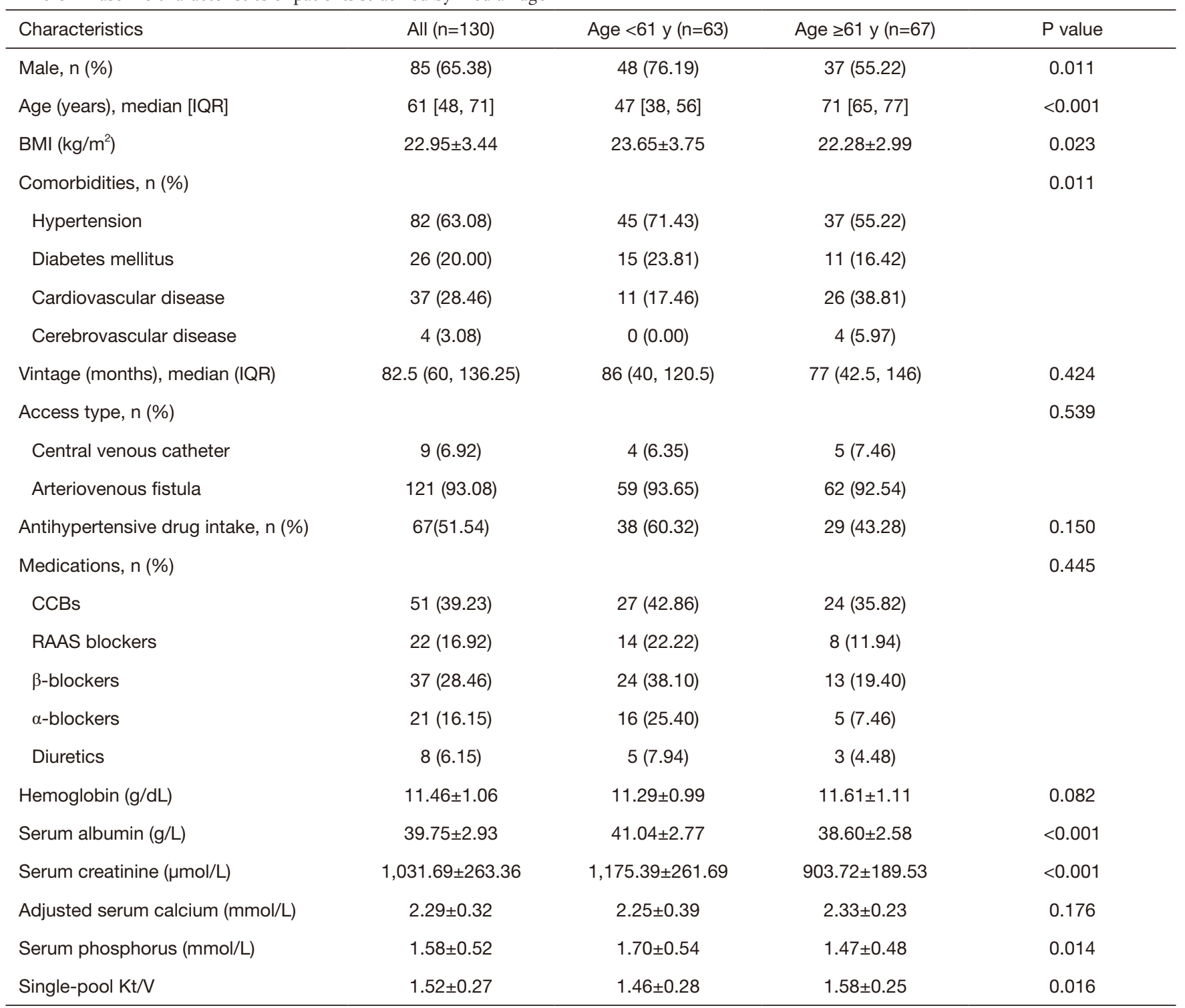

IQR, interquartile range; BMI, body mass index; CCB, calcium channel blocker; RAAS, renin-angiotensin-aldosterone system.

A comparison of parameters between subgroups before and during H-GMQ was shown in Table 4. In the younger group, both the pre-dialysis SBP and DBP were elevated significantly during H-GMQ, neither SBP nor DBP changed significantly in the elderly group. Both groups had increased HR and declined serum sodium. The IDWGR increased during H-GMQ in the elderly group.

\section{Discussion}

Our study showed that the 2-week H-GMQ increased the pre-dialysis BP in MHD patients, the effect was more pronounced in patients less than 61 years old.

The median incubation period for COVID-19 was 5.1 days, about $97.5 \%$ of people infected with COVID-19 would develop symptoms within 11.5 days of exposure (5), suggesting that a 14-day quarantine period for individuals with likely exposure to the coronavirus was reasonable. It was reported that MHD patients were highly susceptible to COVID-19 and HD centers were high-risk area (6). Therefore, all the HD patients exposed to COVID-19 in our center were required to be under H-GMQ in separate 
Table 2 Clinical, laboratory and mental health changes between baseline and H-GMQ ( $\mathrm{n}=130)$

\begin{tabular}{lccc}
\hline Variables & Baseline & H-GMQ & P value \\
\hline SBP $(\mathrm{mmHg})$ & $153.00 \pm 17.96$ & $156.99 \pm 17.46$ & $<.001$ \\
DBP $(\mathrm{mmHg})$ & $76.56 \pm 15.21$ & $77.52 \pm 12.44$ & 0.271 \\
MAP $(\mathrm{mmHg})$ & $101.80 \pm 11.92$ & $103.99 \pm 11.40$ & 0.001 \\
Intra-dialytic SBPV $(\mathrm{mmHg})$ & $12.25 \pm 3.79$ & $12.06 \pm 3.54$ & 0.559 \\
HR $(\mathrm{bpm})$ & $74.44 \pm 10.13$ & $78.38 \pm 10.40$ & $<0.001$ \\
IDWGR $(\%)$ & $2.46 \pm 0.76$ & $2.51 \pm 0.70$ & 0.182 \\
UFR (mL/kg/h) & $9.93 \pm 7.42$ & $8.93 \pm 3.03$ & 0.377 \\
Serum sodium (mmol/L) & $139.31 \pm 3.17$ & $137.57 \pm 3.03$ & $<0.001$ \\
Serum potassium (mmol/L) & $4.74 \pm 0.65$ & $4.70 \pm 0.64$ & 0.517 \\
SAS & $40.11 \pm 9.62$ & $41.24 \pm 8.84$ & 0.382 \\
SDS & $46.85 \pm 10.85$ & $47.52 \pm 11.83$ & 0.376 \\
\hline
\end{tabular}

The values of SBP, DBP, MAP, intra-dialytic SBPV, HR, IDWGR, and UFR were the mean values of the 2 weeks. H-GMQ, hospital-based group quarantine; SBP, systolic blood pressure; DBP, diastolic blood pressure; MAP, mean arterial pressure; SBPV, systolic blood pressure variability; HR, heart rate; IDWGR, inter-dialytic weight gain rate; UFR, ultrafiltration rate; SAS, self-rating anxiety scale; SDS, self-rating depression scale.

Table 3 Univariate and multivariate analyses for change in pre-dialysis SBP ( $\mathrm{n}=130)$

\begin{tabular}{|c|c|c|c|c|}
\hline Variables & \multicolumn{2}{|c|}{ Univariate, Pearson's or Spearman's correlation } & \multicolumn{2}{|c|}{ Multivariate, linear regression model } \\
\hline Gender (male) & 0.079 & 0.372 & 0.203 & 0.128 \\
\hline Age (years) & -0.229 & 0.009 & -0.324 & 0.014 \\
\hline Hypertension & 0.229 & 0.009 & 0.205 & 0.144 \\
\hline Dialysis vintage (months) & 0.062 & 0.486 & -0.062 & 0.648 \\
\hline $\mathrm{BMI}\left(\mathrm{kg} / \mathrm{m}^{2}\right)$ & -0.082 & 0.353 & -0.222 & 0.088 \\
\hline Difference in HR (bpm) & 0.139 & 0.114 & -0.009 & 0.946 \\
\hline Difference in IDWGR (\%) & -0.074 & 0.405 & 0.009 & 0.941 \\
\hline Difference in SAS & 0.174 & 0.143 & 0.051 & 0.725 \\
\hline Difference in SDS & 0.049 & 0.678 & -0.043 & 0.773 \\
\hline
\end{tabular}

The difference was defined as value during quarantine minus before; $r$ means Pearson's or Spearman's correlation coefficients; $\beta$ means standardized regression coefficients. SBP, systolic blood pressure; BMI, body mass index; HR, heart rate; IDWGR, inter-dialytic weight gain rate; SAS, self-rating anxiety scale; SDS, self-rating depression scale.

rooms and received inpatient care for 14 days. Fortunately, no new COVID-19 cases emerged during the H-GMQ period.
The biggest changes of these 14 days were their living environment and lifestyle. We speculated that the changes in lifestyle may result in changes in sodium intake, 
Table 4 Clinical, laboratory and mental health changes between baseline and H-GMQ stratified by age

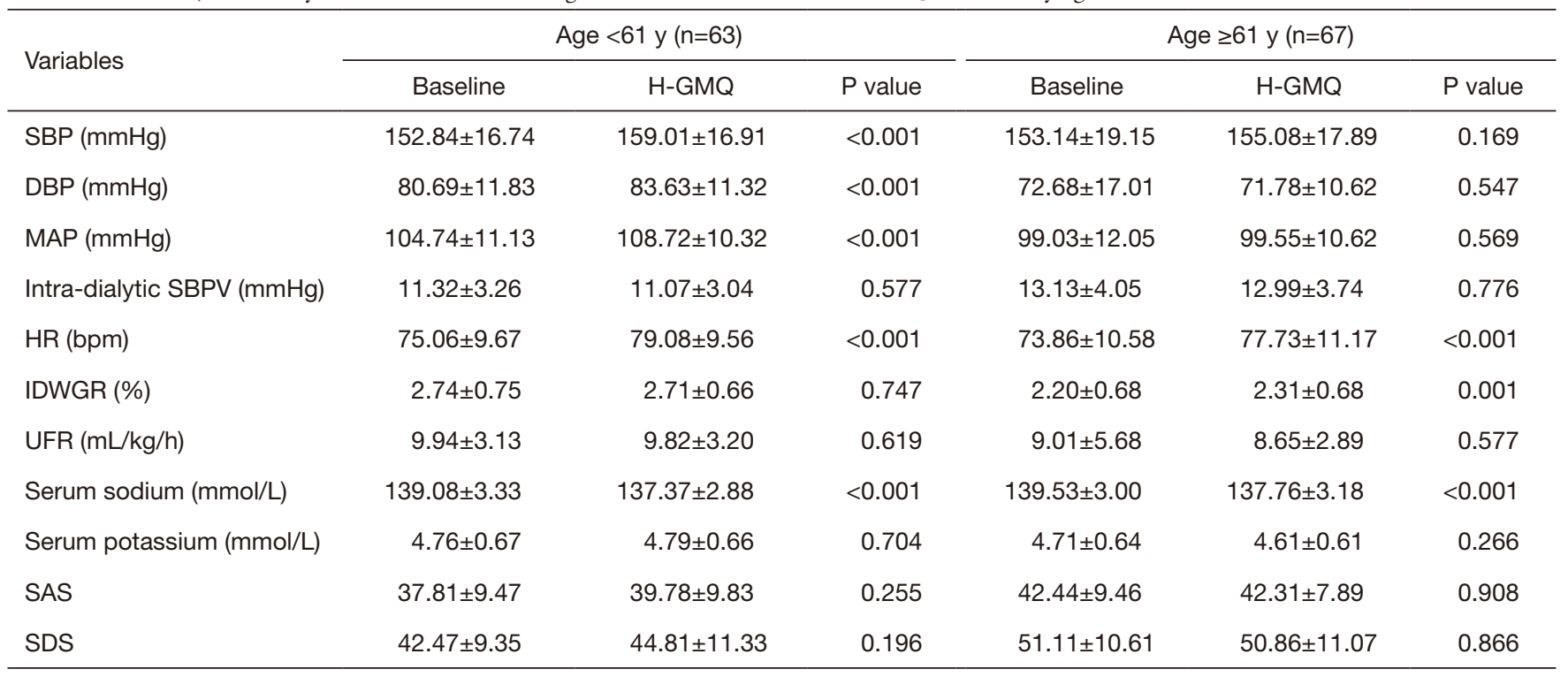

The values of SBP, DBP, MAP, intra-dialytic SBPV, HR, IDWGR, and UFR were the mean values of the 2 weeks. H-GMQ, hospital-based group quarantine; SBP, systolic blood pressure; DBP, diastolic blood pressure; MAP, mean arterial pressure; HR, heart rate; SBPV, systolic blood pressure variability; IDWGR, inter-dialytic weight gain rate; UFR, ultrafiltration rate; SAS, self-rating anxiety scale; SDS, self-rating depression scale.

extracellular volume, and even mental health, which would, in turn, affect their BP. For example, the diet diary showed that the mean dietary sodium intake during quarantine was $3.3 \mathrm{~g} / \mathrm{d}$, which meant the patients took $8.3 \mathrm{~g} / \mathrm{d}$ of salt. Since the average serum sodium declined by nearly $2 \mathrm{mmol} / \mathrm{L}$ after H-GMQ, and the dialysate sodium concentration remained unchanged, it's reasonable to speculate that although exceeding the recommendation dose, they still took less sodium during quarantine than before. However, we knew that sodium restriction was linked to extracellular volume reduction and decrease of vascular smooth muscle cell tone, resulting in BP decrease instead of increase (7). Moreover, no significant difference in IDWG and SAS/ SDS score was observed before and during H-GMQ, which appeared to contradict our hypothesis.

We proposed several possible explanations for these paradoxical findings. First, based on findings after the outbreak of MERS, HD patients under H-GMQ showed a good level of adjustment and had low rates of reporting psychological distress (8), which was similar to our findings. It seemed that maybe the quarantined patients wanted to receive HD and inpatient care in the first place, or the timely communication with medical staffs ameliorated their anxiety and depression. Second, the paradoxical relationship between serum sodium and BP could be explained by the delayed response of BP. It is known that reduction of the extracellular volume does not translate into an immediate reduction in BP, but may take weeks to months, which is called the "lag phenomenon" (9). Maybe the 2-week change in diet was just long enough for us to observe the decline of serum sodium but not BP. Another explanation was that other variables elevated $\mathrm{BP}$ so much that counteracted the effect of serum sodium, for example, the increased activity of the sympathetic nervous system. Although no substantial evidence, such as the plasma adrenaline and noradrenaline activity, or the sympathetic nerve activity (SNA) was available, those patients' HR did increase significantly during H-GMQ, which was a sign of sympathetic overactivity.

Also, our study showed that age had a negative correlation with BP elevation, which meant the elderly had a more stable pre-dialysis BP than younger patients. It was usually believed that aging was associated with increased SNA and loss of the balancing factors seen in younger people, leading to dysfunction of BP regulation in older people (10). This result reminded us that either the balancing mechanisms of BP regulation were lost in young MHD patients, or they got more nervous than the elderly, indicating psychological support should be provided for those under quarantine. 
The major strength of this study is that there is no published report on quarantining such a large cohort of MHD patients. As the COVID-19 pandemic is still spreading, the results of this study may provide useful information for HD centers around the world to cope with similar situations.

There are also some limitations of this study. First, this is a prospective observational study, the mechanism underlying our findings are not fully elucidated. Second, this study is limited to the COVID-19 outbreak, other potential variables, such as social-economic issues are not considered.

\section{Conclusions}

In conclusion, our study found that H-GMQ may increase the pre-dialysis BP of MHD patients, especially in younger patients. More attention should be paid to these patients' $\mathrm{BP}$ if another quarantine is needed.

\section{Acknowledgments}

We thank all the colleagues involved in this H-GMQ program. We also want to thank our patients and their family for their understanding and cooperation.

Funding: This work was supported by Capital Clinical Characteristics Application Program of Beijing Municipal Science and Technology Commission (No. Z131107002213122). The funder has no role in the preparation of data or the manuscript.

\section{Footnote}

Reporting Checklist: The authors have completed the STROBE reporting checklist. Available at http://dx.doi. org/10.21037/apm-20-1421

Data Sharing Statement: Available at http://dx.doi. org/10.21037/apm-20-1421

Conflicts of Interest: All authors have completed the ICMJE uniform disclosure form (available at http://dx.doi. org/10.21037/apm-20-1421). The authors have no conflicts of interest to declare.

Ethical Statement: The authors are accountable for all aspects of the work in ensuring that questions related to the accuracy or integrity of any part of the work are appropriately investigated and resolved. This study was approved by the Ethics Committee of Peking University People's Hospital (ethical approval number: 2020PHB04101). All procedures performed in this study involving human participants were in accordance with the Declaration of Helsinki. Patients had given their informed written consent before study entry.

Open Access Statement: This is an Open Access article distributed in accordance with the Creative Commons Attribution-NonCommercial-NoDerivs 4.0 International License (CC BY-NC-ND 4.0), which permits the noncommercial replication and distribution of the article with the strict proviso that no changes or edits are made and the original work is properly cited (including links to both the formal publication through the relevant DOI and the license). See: https://creativecommons.org/licenses/by-nc-nd/4.0/.

\section{References}

1. Harapan H, Itoh N, Yufika A, et al. Coronavirus disease 2019 (COVID-19): a literature review. J Infect Public Health 2020;13:667-73.

2. Saran R, Robinson B, Abbott KC, et al. US Renal Data System 2016 Annual Data Report: epidemiology of kidney disease in the United States. Am J Kidney Dis 2017;69:A7-8.

3. Hu Y, Sun S, Gang L, et al. Cross-sectional investigation of the intradialytic blood pressure variability and its related factors in maintenance hemodialysis patients. Chin J Blood Purif 2019;18:531-4.

4. Zung WW. A rating instrument for anxiety disorders. Psychosomatics 1971;12:371-9.

5. Lauer SA, Grantz KH, Bi Q, et al. The Incubation Period of Coronavirus Disease 2019 (COVID-19) from publicly reported confirmed cases: estimation and application. Ann Intern Med 2020;172:577-82.

6. Ma Y, Diao B, Lv X, et al. 2019 novel coronavirus disease in hemodialysis (HD) patients: Report from one HD center in Wuhan, China. medRxiv 2020. doi: 10.1101/2020.02.24.20027201.

7. Hörl MP, Hörl WH. Hemodialysis-associated hypertension: pathophysiology and therapy. Am J Kidney Dis 2002;39:227-44.

8. Lee SM, Kang WS, Cho AR, et al. Psychological impact of the 2015 MERS outbreak on hospital workers and quarantined hemodialysis patients. Compr Psychiatry 2018;87:123-7. 
9. Khosla UM, Johnson RJ. Hypertension in the hemodialysis patient and the "lag phenomenon": insights into pathophysiology and clinical management. Am J Kidney Dis 2004;43:739-51.

Cite this article as: Wang Y, Gan L, Zuo L, Yang B, Wang Y, Liang J, Ni M. Hospital-based group medical quarantine increased the pre-dialysis blood pressure in maintenance hemodialysis patients. Ann Palliat Med 2021;10(2):1920-1927. doi: 10.21037/apm-20-1421
10. Briant LJ, Charkoudian N, Hart EC. Sympathetic regulation of blood pressure in normotension and hypertension: when sex matters. Exp Physiol 2016;101:219-29. 